\title{
Automatic Adductors Angle Measurement for Neurological Assessment of Post-neonatal Infants during Follow Up
}

\author{
Debi Prosad Dogra ${ }^{1}$, Arun Kumar Majumdar ${ }^{1}$, Shamik Sural ${ }^{1}$, \\ Jayanta Mukherjee $^{1}$, Suchandra Mukherjee ${ }^{2}$, and Arun Singh ${ }^{2}$ \\ 1 IIT Kharagpur, India, 721302 \\ \{dpdogra, akmj, shamik, jay\}@cse.iitgp.ernet.in \\ 2 IPGMER and SSKM Hospital, Kolkata, India, 700020 \\ drsmukherjee71@gmail.com, drarunsingh61@yahoo.co.in
}

\begin{abstract}
In many medical examinations, image or video based automatic schemes are preferred over conventional approaches. Such schemes can greatly increase the efficacy and accuracy of various medical examinations. The work proposed in this article presents an image processing based method to automate adductors angle measurement which is carried out on infants as a part of Hammersmith Infant Neurological Examination (HINE). It is used for assessing neurological development of infants aged below two years. During HINE, postures and reactions of the infant under consideration are recorded. An overall score is estimated and used to quantify the neurological development index of the baby. In the conventional approach, for measuring adductors angle, doctors use rulers. The proposed method uses image segmentation and thinning techniques to measure the angle without involvement of rulers. Results show that the proposed scheme can be used as an aid to the doctors for conducting such examinations.
\end{abstract}

Keywords: Adductors Angle, Skeleton Generation, Image Segmentation, Neurological Assessment.

\section{Introduction}

Nowadays, computer vision based techniques are frequently used to improve various health-care services. Sophisticated instruments equipped with state-ofthe-art video technologies are widely used in conducting medical examinations and tele-consultations. For example, video conferencing based tele-medicine applications, automatic analysis of X-ray, MRI, CT images and video EEG signals are popular in medical community. These technologically advanced tools can significantly improve the average turnaround time of examinations.

A widely used neurological examination which is conducted on infants of less than two years of age is the Hammersmith Infant Neurological Examination (HINE) 6]. The examination is carried out repetitively at the ages of 3, 6, 9, 12 months and onwards. An overall score is estimated to quantify the neurological development of the baby at the time of examination. The score is estimated using examination outcomes which are broadly classified into three groups namely, 
Neurological Items, Motor Milestones and Behavior. Among these three, the first group deals with the neurological development and it has five subgroups named as, cranial nerve function, postures, movements, tone, reflexes and reactions. According to HINE specification, all examinations under these subgroups are quantified by scores between zero to three. A score of three signifies that the development of the baby is normal and zero means abnormal. The remaining two groups deal with motor and behavioral development. There is no such standard scoring scheme available for motor development whereas behavioral development is a subjective evaluation [6].

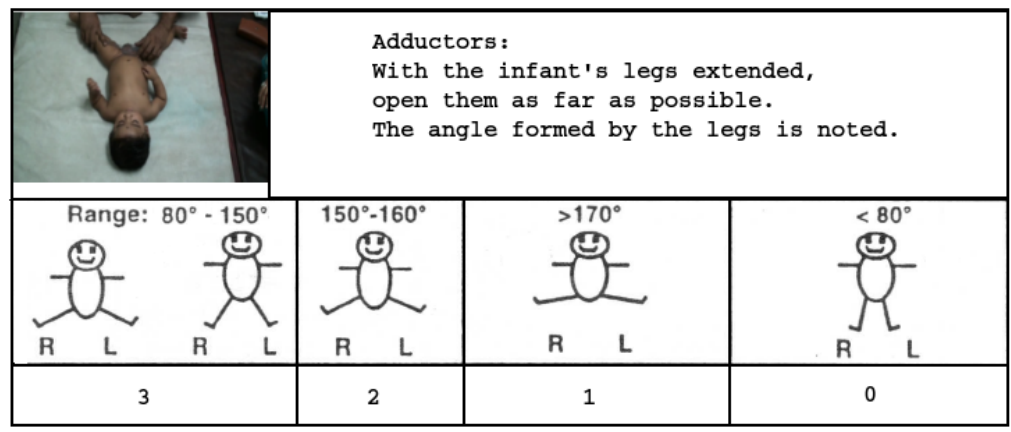

Fig. 1. Sample image of conventional adductors angle measurement with reference templates and scores. The image was captured at the HINE clinic of the Neonatology department of IPGMER and SSKM Hospital, Kolkata, India.

In order to have accurate estimation of neurological parameters, video and image processing based algorithms can be developed to facilitate estimation of parameters like adductors, popliteal angle, ankle dorsiflexion, arm protection, pulled-to-sit, etc. Though, several applications in medical informatics domain adopt computer vision based approaches, none of them attempted to automate HINE. For example, Bhatt et al. 2] have proposed a video based scheme for recognizing gestures of baby to automatically detect accidental events. Similarly, the application developed by Singh et al. [15] is a tele-monitoring system for remote surveillance of infants. It was developed using safe, compact and non-invasive sensors to record the movements of a baby with a client / server based approach. Similar kinds of applications are also found in health-care domain which adopt computer vision based techniques. For example, a simulation based infant behavior analysis using a virtual environment is proposed in [14] where the authors have tried to avoid situations related to behavioral accidents. The authors of 4 have proposed an application that can be used to record the videos and patient details during HINE. However, there is no work reported in the literature that tries to automate the examinations of HINE through image or video processing based algorithms. Therefore, till date, doctors conduct and record the outcomes of HINE manually.

In the chart proposed by Dubowitz et al. 6], there are a few examinations which are related to angle measurement of various human body parts. We have selected one of those examinations for automation. The examination is called 
as adductors angle measurement. In Fig. 1, a sample image of the scene during adductors angle measurement is shown with reference templates and scores. It has been observed that during examinations involving measurement of various angles, especially adductors angle and popliteal angle, the examiner often finds it difficult to keep the baby in a stable state. Since the baby moves frequently, placing a ruler across body parts for angle measurements become difficult. In addition to that, often the baby starts showing sign of uneasiness during these examinations. This has motivated us to design image processing based algorithms to help the doctors during angle measurement examinations.

\section{Proposed Angle Measurement Scheme}

During adductors examination, the angle between the thighs of the infant at supine posture is measured and a score is assigned according to the reference templates (refer to Fig. 1). In the conventional approach, the examiner stretches the legs of the infant and rulers are used to measure the angle between the thighs as shown in the figure. An image based automatic scheme is proposed in this work. After surveying the HINE clinic of the Neonatology department of Institute of The PostGraduate Medical Education \& Research (IPGMER) and Seth Sukhlal Karnani Memorial (SSKM) Hospital, Kolkata, India, it was observed that a single camera is sufficient to capture the scene of the examination. The examination desk has been covered with soft mattress. This was done in accordance with the well established setup of the HINE clinic of the hospital. Normally, all clothings are removed from the baby before examination commences. Since the measurement of the adductors angle is recorded only when the expert feels first resistance from the infant while stretching the legs, we have captured one image per baby.

\subsection{Segmentation and Binarization of Image}

Estimation of adductors angle can be automated using the following image processing steps. First, a color based segmentation algorithm is used to distinguish between the background and foreground parts. Since the background is controlled, a color based separation is possible. We have tested with various popular image segmentation schemes that are commonly used for natural image segmentation [3], 8] - 11. Performance of mean-shift based segmentation scheme proposed by Comaniciu et al. 3] for the images of the domain under consideration has been found to be satisfactory. Also, the authors of [5] and [12] have shown in their work that mean-shift outperforms other algorithms in terms of quality. After segmentation, small and insignificant regions are merged with surrounding regions of larger size and importance (see Fig. 2b). To separate the foreground object (infant at supine and hands of the examiner) from the background, region that is dominated by human skin color is selected. A binary image is produced where the black pixels represent foreground and white pixels represent background (see Fig. 26).

\subsection{Skeletonization of Binary Object}

Next, a widely used thinning scheme based on safe point thinning algorithm (SPTA) 13 is applied on the foreground objects. SPTA iteratively removes the 
boundary points of the object from all directions and finally produces a thinned object. Some examples of thinned objects are shown in Fig. 2 d. Since the thinning algorithm produces noisy outputs, measurement of angle between the legs of the infant using these outputs is difficult. Thus, for better estimation, a filtering process has been proposed which successfully removes insignificant edges and produces usable skeleton image of the baby.

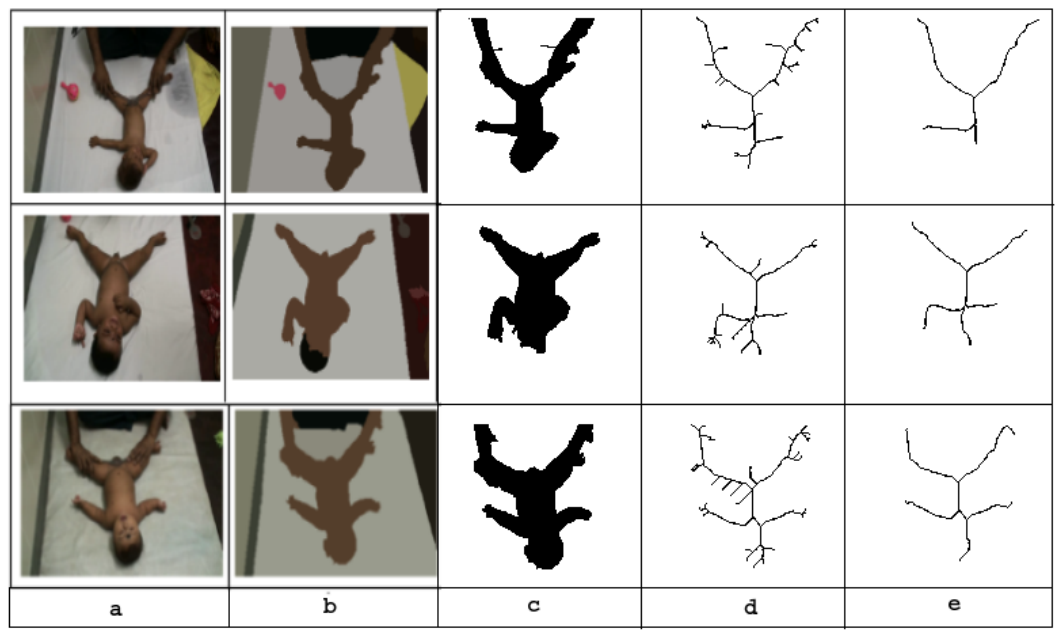

Fig. 2. a. Original images b. Segmented images using mean-shift [3] and region merging with spatial bandwidth $\left(h_{s}\right)=7$ and range bandwidth $\left(h_{r}\right)=12.5$ and minimum region area $=400$ pixels c. Binarized outputs d. SPTA based thinning [13] e. Outputs after proposed filtering using $T=0.1$.

\subsection{Filtering}

Let, the thinned object generated using the SPTA algorithm be represented by a graph $G(V, E)$ where the segments of the skeleton are considered as graph edges and joining or end points of the segments are denoted by nodes. An edge is marked as a hanging edge when one of its vertices is a leaf node. Set of hanging edges is denoted by $H$ where $H \subseteq E$. Next, a greedy algorithm is adopted that iteratively removes the smallest edge out of all edges hanging from an intermediate vertex (see Algorithm 1). The algorithm is capable of removing small and insignificant hanging edges and produces a filtered output $G\left(V^{\prime}, E^{\prime}\right)$. The filtering steps are elaborated with a synthetic example shown in Fig. 4. Intermediate nodes and the leaf nodes are colored differently for better visualization. The proposed scheme produces filtered skeleton images as shown in Fig. 22. Using these filtered graphs, adductors angle can be measured. The threshold $T$ as mentioned in the filtering algorithm is normally set to $10 \%$ of the largest edge of the entire graph. 


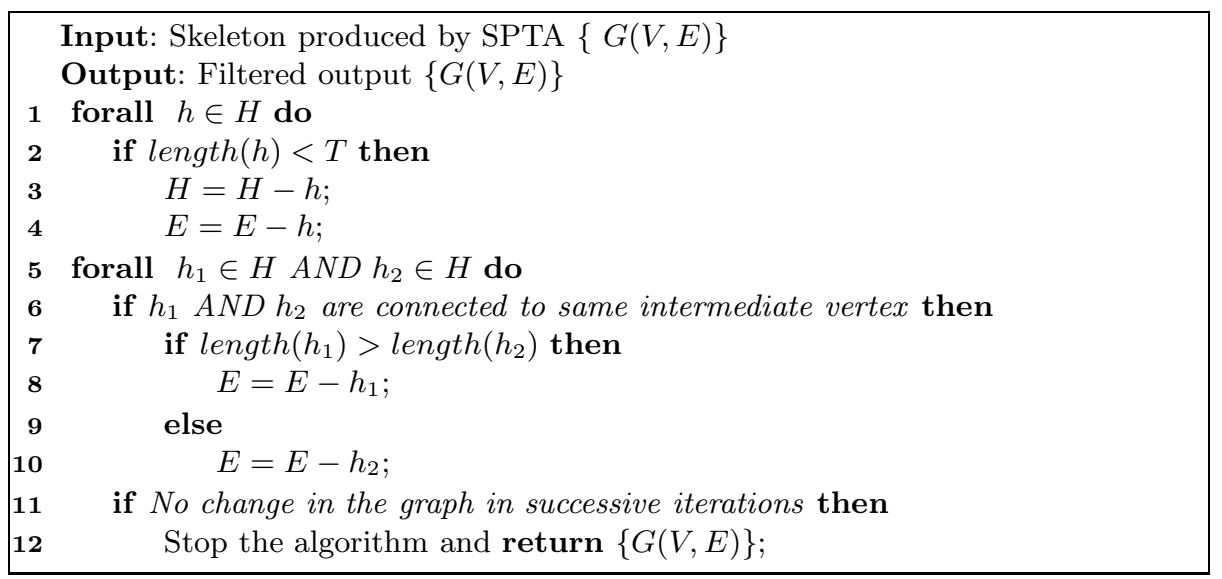

Algorithm 1. Filtering after SPTA based skeletonization

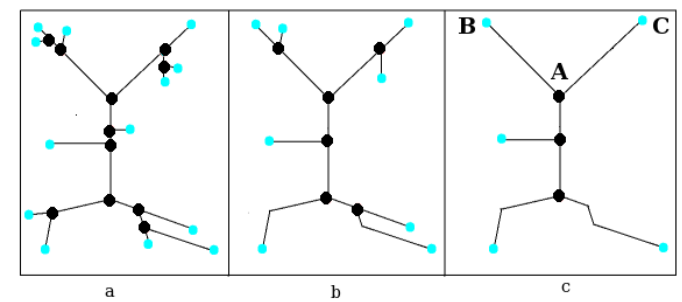

Fig. 3. Outputs at various intermediate steps of the filtering process a. Initial skeleton b. Intermediate skeleton c. Final skeleton.

\subsection{Adductors Angle Measurement}

The reduced graph generated by Algorithm 1 is used for estimating adductors angle. In the graph, we search for the intermediate node A of $G\left(V^{\prime}, E^{\prime}\right)$ as shown in Fig. 3r. It is expected to be the joining point between two thighs since the baby is placed supine with an upside down view with respect to camera. Next, two straight lines are fitted along the edges representing the thighs of the baby using least square approximation method. Finally, the angle between the straight line segments denoted by $\mathrm{AB}$ and $\mathrm{AC}$ is estimated (refer to Fig. 3 .).

\section{Results and Discussions}

We have recorded visuals of adductors angle measurement for 50 babies at the HINE clinic of SSKM hospital. Out of these 50 cases, the proposed filtering algorithm was capable of generating usable skeletons for 46 images. Comparative results using the proposed scheme with respect to ground truths are shown in Fig. 4. We have plotted the ground truths recorded by the experts and angle measured by the proposed scheme in the graph. In the graph, x-axis and $y$-axis denote sample number and angle in degree. It is evident from the graph that the results agree with ground truth measurements (angle measured by doctors) 
for majority of the samples. In the next part of the analysis, classification with respect to ground truth scores is presented (see Table 1).

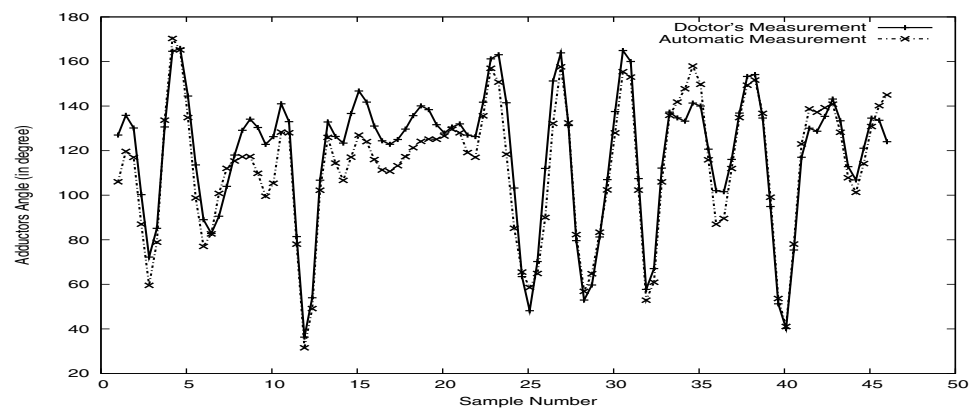

Fig. 4. Comparison of automatic measurement with respect to ground truths recorded during adductors angle measurement at SSKM hospital.

Since the scoring is done based on a range of angles, effect of errors in estimating the scores is less. Thus, high precision and recall is achieved. Fig. 5 shows some results of abductors angle measurement. For better visualization, we have drawn two thick straight lines (colored white) along the thighs of the baby and measured the angle between them.

Table 1. Classification of Results with respect to Recommended Scores of the HINE Chart

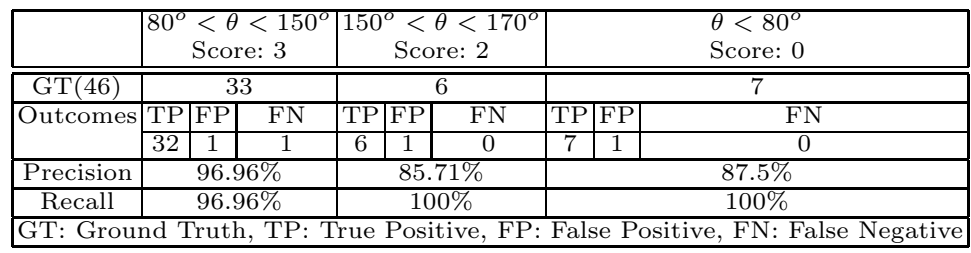

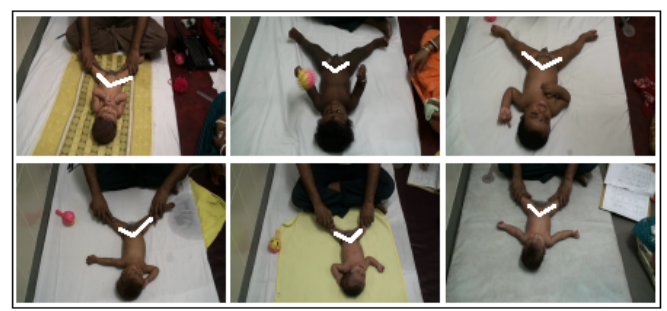

Fig. 5. Results of abductors angle measurement on some of the sample images collected from IPGMER and SSKM Hospital

\section{Conclusion and Future Directions}

In this work, an image processing based algorithm has been proposed that can be used to measure the adductors angle of infants during HINE. This can be used to 
automate the neurological assessment process. Since rulers and other measuring instruments have to be used in a conventional approach, an automated scheme will reduce the workload of the examiner to a considerable extent. Though, in a few cases, our algorithm fails to measure the angle precisely, identifying range of angle instead of exact value is possible with high accuracy. Other examinations like measuring the popliteal angle, curvature in ventral suspension and ankle dorsiflexion can be automated using similar techniques.

\section{Acknowledgement}

The work has been funded by Ministry of Communication and Information Technology, Department of Information Technology, Govt. of India, under Approval No. 1(4)/2009-ME\& TMD (28-08-2009).

\section{References}

1. Arbelaez, P., Maire, M., Fowlkes, C., Malik, J.: From Contours to Regions: An Empirical Evaluation. In: CVPR, pp. 2294-2301 (2009)

2. Bhatt, J., Bebis, G.: Automatic Recognition of a Baby Gesture. In: ICTAI, pp. 610-615 (2003)

3. Comaniciu, D., Meer, P.: Mean Shift: A Robust Approach Toward Feature Space Analysis. IEEE Transactions on Pattern Analysis and Machine Intelligence 24(5), 603-619 (2002)

4. Dogra, D., Nandam, K., Majumdar, A., Sural, S., Mukherjee, J., Mukherjee, S., Singh, A.: A User Friendly Implementation for Efficiently Conducting Hammersmith Infant Neurological Examination. In: Healthcom, pp. 374-378 (2010)

5. Dogra, D.P., Majumdar, A.K., Sural, S.: Evaluation of Segmentation Techniques Using Region Size and Boundary Information. In: Chaudhury, S., Mitra, S., Murthy, C.A., Sastry, P.S., Pal, S.K. (eds.) PReMI 2009. LNCS, vol. 5909, pp. 285-290. Springer, Heidelberg (2009)

6. Dubowitz, L., Dubowitz, V.: The Neurological Assessment of the Preterm and Full Term Infant. Clinics in Developmental Medicine 9 (1981)

7. Felzenszwalb, D.: Efficient Graph-based Image Segmentation. International Journal of Computer Vision 59(2), 167-181 (2004)

8. Kuan, Y., Kuo, C., Yang, N.: Color-Based Image Salient Region Segmentation Using Novel Region Merging Strategy. IEEE Transactions on Multimedia 10(5), 832-845 (2008)

9. Martin, D.: An Empirical Approach to Grouping and Segmentation. Ph.D. Dissertation, Univ. of California, Berkeley (2002)

10. Pal, N.R., Pal, S.K.: A Review on Image Segmentation Techniques. Journal of Pattern Recognition 26(9), 1277-1294 (1993)

11. Shi, J., Malik, J.: Normalized Cuts and Image Segmentation. IEEE Transactions on Pattern Analysis and Machine Intelligence 22(8), 888-905 (2000)

12. Unnikrishnan, R., Pantofaru, C., Hebert, M.: Toward Objective Evaluation of Image Segmentation Algorithms. IEEE Transactions on Pattern Analysis and Machine Intelligence 29(6), 929-944 (2007)

13. Naccache, N., Shinghal, R.: SPTA: A Proposed Algorithm for Thinning Binary Patterns. IEEE Transactions on Systems Man and Cybernatics 14(3), 409-418 (1984)

14. Nishida, Y., Motomura, Y.: Infant Behavior Simulation Based on an Environmental Model and a Developmental Behavior Model. ICSMC 2, 1555-1560 (2004)

15. Singh, S., Hsiao, H.: Infant Telemonitoring System. In: ICEMBS, pp. 1354-1357 (2003) 